\title{
Biosynthesis of Coenzyme Q in the Phytopathogen Xanthomonas campestris via a Yeast-Like Pathway
}

\author{
Lian Zhou, ${ }^{1,2}$ Ming Li, ${ }^{1}$ Xing-Yu Wang, ${ }^{1}$ Hao Liu, ${ }^{1}$ Shuang Sun, ${ }^{1}$ Haifeng Chen, ${ }^{1}$ Alan Poplawsky, ${ }^{3}$ and \\ Ya-Wen $\mathrm{He}^{1,+}$ \\ ${ }^{1}$ State Key Laboratory of Microbial Metabolism, Joint International Research Laboratory of Metabolic \& Developmental \\ Sciences, School of Life Sciences \& Biotechnology, Shanghai Jiao Tong University, Shanghai 200240, China; ${ }^{2}$ Zhiyuan \\ Innovation Research Centre, Student Innovation Centre, Zhiyuan College, Shanghai Jiao Tong University; and ${ }^{3}$ Department of \\ Entomology, Plant Pathology and Nematology, University of Idaho, Moscow 83844, U.S.A.
}

Accepted 26 July 2018.

\begin{abstract}
Coenzyme Q $(\mathrm{CoQ})$ is a lipid-soluble membrane component found in organisms ranging from bacteria to mammals. The biosynthesis of CoQ has been intensively studied in Escherichia coli, where 12 genes (ubiA, $-B,-C,-D,-E,-F,-G,-H,-I,-J,-K$, and $-X$ ) are involved. In this study, we first investigated the putative genes for CoQ8 biosynthesis in the phytopathogen Xanthomonas campestris pv. campestris using a combination of bioinformatic, genetic, and biochemical methods. We showed that Xc_0489 $\left(\operatorname{coq} 7^{X c}\right)$ encodes a di-iron carboxylate monooxygenase filling the $E$. coli $\mathrm{UbiF}$ role for hydroxylation at C-6 of the aromatic ring. Xc_0233 (ubiJ ${ }^{X c}$ ) encodes a novel protein with an $E$. coli UbiJ-like domain organization and is required for CoQ8 biosynthesis. The $X$. campestris pv. campestris decarboxylase gene remains unidentified. Further functional analysis showed that $u b i B$ and $u b i K$ homologs $u b i B^{\mathrm{Xc}}$ and $u b i K^{\mathrm{Xc}}$ are required for CoQ8 biosynthesis in $X$. campestris pv. campestris. Deletion of $u b i J^{\mathrm{Xc}}, u b i B^{\mathrm{Xc}}$, and $u b i K^{\mathrm{Xc}}$ led to the accumulation of an intermediate 3-octaprenyl-4-hydroxybenzoic acid. $\mathrm{UbiK}^{\mathrm{Xc}}$ interacts with $\mathrm{UbiJ}^{\mathrm{Xc}}$ and $\mathrm{UbiB}^{\mathrm{Xc}}$ to form a regulatory complex. Deletion analyses of these CoQ8 biosynthetic genes indicated that they are important for virulence in Chinese radish. These results suggest that the $X$. campestris pv. campestris CoQ8 biosynthetic reactions and regulatory mechanisms are divergent from those of $E$. coli. The variations provide an opportunity for the design of highly specific inhibitors for the prevention of infection by the phytopathogen $X$. campestris pv. campestris.
\end{abstract}

Coenzyme Q (CoQ) is a lipid-soluble membrane component found in organisms ranging from bacteria to mammals. It consists of a conserved aromatic ring and a polyprenyl hydrophobic tail with the number of isoprenyl units varying among

\section{Zhou and M. Li contributed equally to this work.}

${ }^{\dagger}$ Corresponding author: Ya-Wen He; E-mail: yawenhe@sjtu.edu.cn

Funding: This research work was financially supported by the research grants from the National Natural Science Foundation of China (numbers 31471743 and 31772121 to Y.-W. He) and National Key Research and Development Program of China (2016YFE0101000 to L. Zhou).

*The $\boldsymbol{e}$-Xtra logo stands for "electronic extra" and indicates that eight supplementary figures and two supplementary tables are published online.

(c) 2019 The American Phytopathological Society species: six in Saccharomyces cerevisiae, eight in Escherichia coli, and 10 in humans (Aussel et al. 2014b; Bentinger et al. 2010). CoQ is a key component of the electron transport chain that participates in aerobic cellular respiration within the mitochondria of eukaryotes and the plasma membrane of prokaryotes (Alcázar-Fabra et al. 2016; Kawamukai 2016; Sharma et al. 2012).

Biosynthesis of CoQ has been intensively studied in both $E$. coli and $S$. cerevisiae. CoQ8 biosynthesis in $E$. coli involves the 12 genes ubiA, $-B,-C,-D,-E,-F,-G,-H,-I,-J,-K$, and $-X$, and most of these encode enzymes that function to decorate the aromatic ring of the 4-hydroxybenzoate (4-HBA) universal precursor (Aussel et al. 2014b; Loiseau et al. 2017). The first defined step in CoQ8 biosynthesis is catalyzed by the $u b i C$ encoded chorismate-pyruvate lyase to produce 4-HBA (Supplementary Fig. S1). UbiA in E. coli subsequently catalyzes the prenylation of 4-HBA with the side-chain precursor octaprenyl PPi (Okada et al. 1997). Then, following condensation, the benzoate ring is modified by decarboxylation, C-hydroxylations, O-methylations, and C-methylation. Decarboxylation of the 3octaprenyl-4-hydroxybenzoic acid (OHB) precursor is catalyzed by the decarboxylase encoded by $u b i D$ and an additional gene, $u b i X$. UbiD is solely responsible for the decarboxylase activity and requires a new type of cofactor: a prenylated flavin synthesized by the associated UbiX, which is a flavin prenyltransferase (Payne et al. 2015; White et al. 2015). Subsequent methylation reactions are catalyzed by the methyltransferases encoded by $u b i G$ and $u b i E$. UbiG is an S-adenosylmethioninedependent methyltransferase, which catalyzes the two Omethylation steps (Poon et al. 1999; Wu et al. 1992), and UbiE is required for C-methylation in CoQ8 biosynthesis (Lee et al. 1997). Three hydroxylation reactions in the biosynthetic pathway introduce hydroxyl groups at positions C-1, C-5, and C-6 of the CoQ8 aromatic ring. $\mathrm{UbiH}$ and $\mathrm{UbiF}$ are proposed flavincontaining monooxygenases responsible for hydroxylation of C- 1 and C- 6 of the aromatic ring. Recent findings demonstrated that the $\mathrm{C} 5$-hydroxylation reaction was catalyzed by the UbiI protein (Hajj Chehade et al. 2013). The role of UbiB remains to be established in E. coli. Human mitochondrial ADCK3, the ortholog of UbiB, was shown to employ an atypical protein kinase-like fold to enable CoQ biosynthesis (Stefely et al. 2015). In addition, it was recently demonstrated that two new genes, $u b i J$ and $u b i K$ (formerly yig $P$ and yqiC, respectively, in E. coli), are required for efficient CoQ biosynthesis (Aussel et al. 2014a; Loiseau et al. 2017). UbiJ may serve as a carrier of the isoprenoid hydrophobic tail prior to the action of monooxygenases and methyltransferases or, alternatively, it could chaperon prenylated 
intermediates during the aerobic hydroxylation steps (Aussel et al. 2014a). Instead of being involved in a specific biosynthetic step, UbiK contributes to global CoQ8 biosynthesis by forming a complex with the C-terminal part of UbiJ (Loiseau et al. 2017).

Xanthomonas spp. are widely distributed, Gram-negative, aerobic bacteria that infect nearly 400 different plant hosts, many of which are commercially important crops such as rice, cotton, soybean, wheat, and vegetables (Leyns et al. 1984). Xanthomonas campestris pv. campestris, the causal agent of crucifer black rot, and $X$. oryzae pv. oryzae, the rice bacterial blight agent, are both model phytopathogens in molecular pathology (Mansfield et al. 2012). Our previous results showed that $X$. campestris pv. campestris and $X$. oryzae pv. oryzae produce CoQ8 as the major ubiquinone (Zhou et al. 2013a and b). Interestingly, both $X$. campestris pv. campestris and $X$. oryzae pv. oryzae contain no homologs of the E. coli chorismate lyase-encoding ubiC (Zhou et al. 2013a and b). Instead, they contain the gene xanB2 (Xcc4014 in X. campestris pv. campestris and PXO_3739 in X. oryzae pv. oryzae), which encodes a novel bifunctional chorismatase needed for the biosynthesis of both 4-HBA and 3-HBA. The XanB2dependent 4-HBA is required for CoQ8 biosynthesis (Zhou et al. $2013 \mathrm{a}$ and $\mathrm{b}$ ). The other genes involved in $X$. campestris pv. campestris CoQ8 biosynthetic pathway remain to be elucidated.

\section{RESULTS}

\section{Bioinformatics analysis}

of $X$. campestris pv. campestris genome to identify the putative genes for CoQ8 biosynthesis.

CoQ8 is the major ubiquinone produced in both $X$. campestris pv. campestris and E. coli. In E. coli, the 12 genes required for CoQ8 biosynthesis are distributed throughout the genome, including the three gene clusters ubiC-ubiA, ubiE$u b i J-u b i B$, and $u b i H-u b i I$ (Fig. 1A). Using these genes as templates, we identified seven E. coli homologs (Xc_0402 for ubiA,

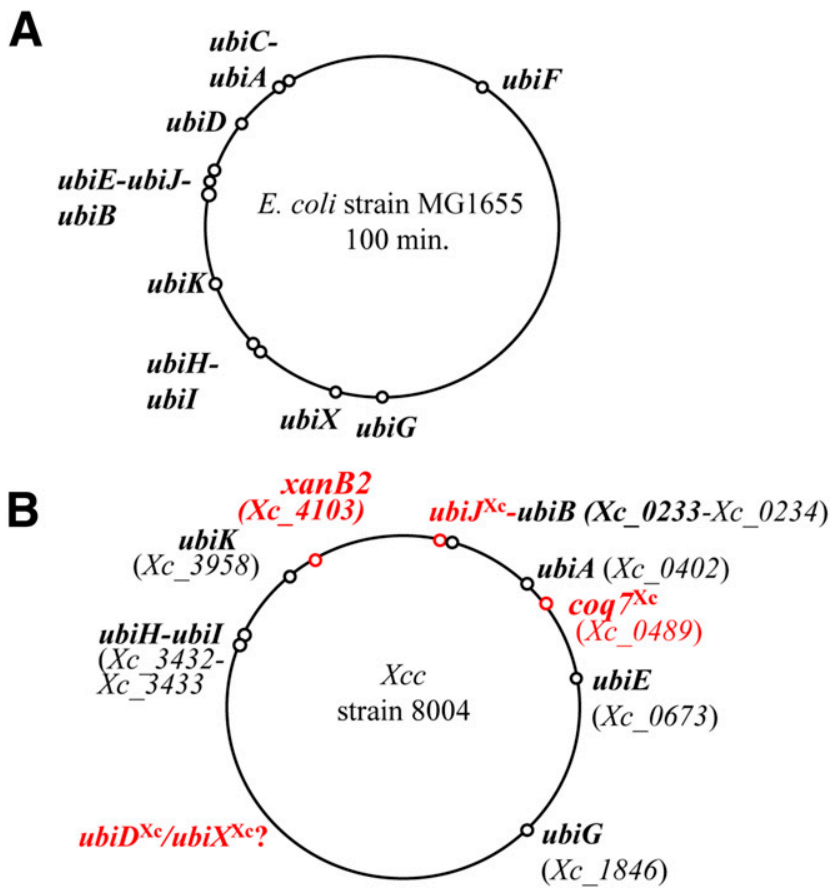

Fig. 1. Genetic organization and location of coenzyme Q8 (CoQ8) biosynthetic genes on the chromosome of A, Escherichia coli strain MG1655 and B, Xanthomonas campestris pv. campestris strain 8004. Genes indicated in a lighter shade are $X$. campestris pv. campestris-specific genes required for CoQ8 biosynthesis. Counterparts of E. coli $u b i D$ and $u b i X$ in $X$. campestris pv. campestris remain unclear.
$X c 0234$ for $u b i B, X c 0673$ for $u b i E, X c \_1846$ for $u b i G$ $X c \_3432$ for $u b i H, X c 3433$ for $u b i I$, and $X c \_3958$ for $\left.u b i K\right)$ in $X$. campestris pv. campestris strain 8004 (Fig. 1B; Supplementary Fig. S2). Only two gene clusters, ubiH (Xc_3432)-ubiI (Xc_3433) and Xc_0233-ubiB (Xc_0234), were observed in $X$. campestris pv. campestris strain 8004 (Fig. 1B). Deletion of $X$. campestris pv. campestris ubiH or ubiI completely abolished CoQ8 biosynthesis (Supplementary Fig. S3). Because we were unable to obtain deletion mutants for ubiA (Xc_0402), ubiG (Xc_1846), or $u b i E$ (Xc_0673), functional copies of these genes are probably required for $X$. campestris pv. campestris growth.

\section{Xc_0489 is required for CoQ8 biosynthesis \\ in $X$. campestris pv. campestris.}

E. coli UbiF is a flavin-containing C6-monooxygenase with a conserved Rossmann-fold NAD(P)H/NAD(P)-binding (NADB) domain (Fig. 2A). Global domain analysis using the program SMART (Letunic et al. 2015) failed to identify an X. campestris pv. campestris gene encoding the UbiF-like NADB domain. Instead, we identified Xc_0489, which encodes a di-iron-containing four-helix-bundle domain typical of a ferritin-like superfamily (Fig. 2A). A similar ferritin-like domain is found in Coq7 of $S$. cerevisiae, another model organism for CoQ biosynthesis (Kawamukai 2016). Xc_0489 shares 35\% amino-acid identity with CoQ7 in yeast (Supplementary Fig. S4) and $47.0 \%$ amino-acid identity with Coq7's homolog PA0655 in Pseudomonas aeruginosa PAO1 (Stenmark et al. 2001). Coq7 is a di-iron enzyme that catalyzes monooxygenation in $S$. cerevisiae CoQ6 biosynthesis (Marbois and Clarke 1996). These findings prompted us to determine whether Xc_0489 (named $\operatorname{coq} 7^{X_{c}}$ hereafter) has CoQ7-like C6-monooxygenase activity needed for $X$. campestris pv. campestris CoQ8 biosynthesis.

$\operatorname{coq} 7^{\mathrm{Xc}}$ was deleted from $X$. campestris pv. campestris wildtype (WT) strain 8004 , and the resulting strain $\Delta \operatorname{coq} 7^{\mathrm{Xc}}$ displayed growth characteristics similar to those of strain 8004 in Luria-Bertani (LB) medium (Fig. 2B). The CoQ8 levels in $\Delta \operatorname{coq} 7^{\mathrm{Xc}}$ cells at $24 \mathrm{~h}$ after inoculation were $26.6 \%$ of those found in WT cells. This CoQ8 deficiency could be restored by the overexpression of $\operatorname{coq} 7^{\mathrm{Xc}}$ via the vector pBBR-1-MCS (kanamycin resistant $\left[\mathrm{Kan}^{\mathrm{R}}\right]$ ) (Fig. 2C). An unknown electroactive compound, denoted " $\mathrm{X}$ ", was detected in the $\Delta \operatorname{coq} 7^{\mathrm{Xc}}$ cells but not in the WT cells. Complementation by $\operatorname{coq} 7^{\mathrm{Xc}}$ abolished the accumulation of compound X (Fig. 2C and D).

In previous work, deletion of $u b i F$ from $E$. coli strain MG1655 resulted in the accumulation of demethoxy-Q8 (DMQ8) (Kwon et al. 2000); thus, we used E. coli strain MG1655 to generate the $u b i F$ deletion strain $\triangle u b i F$ and confirmed the DMQ8 accumulation (Supplementary Fig. S5). Further comparison showed that compound $\mathrm{X}$ produced by $X$. campestris pv. campestris mutant $\Delta \operatorname{coq} 7^{\mathrm{Xc}}$ had the same elution time and absorbance maximum as DMQ8 from $E$. coli mutant $\triangle u b i F$. Subsequent ultrahigh-performance liquid chromatography mass spectrometry (UPLC-MS) analysis verified the identity of compound X as DMQ8 (Fig. 2E).

Overexpression in strain $\Delta \operatorname{coq} 7^{\mathrm{Xc}}$ of either E. coli $u b i F$ or S. cerevisiae $\operatorname{coq} 7$ via the vector $\mathrm{pBBR}-1-\mathrm{MCS}\left(\operatorname{Kan}^{\mathrm{R}}\right)$ partially restored CoQ8 production (Fig. 3B). Conversely, overexpression of $\operatorname{coq} 7^{\mathrm{Xc}}$ in the $E$. coli strain $\Delta u b i F$ restored CoQ8 production to WT levels (Fig. 3D). Collectively, these results demonstrate that $\operatorname{coq} 7^{\mathrm{Xc}}$ is homolog of $S$. cerevisiae $\operatorname{coq} 7$, playing the same role as E. coli ubiF in X. campestris pv. campestris CoQ8 biosynthesis.

\section{$\operatorname{Coq} 7^{\mathrm{Xc}}$ is a di-iron carboxylate monooxygenase} with a four-helix bundle.

The $S$. cerevisiae Coq7 protein contains the conserved di-ironbinding motif $\mathrm{EX}_{\mathrm{n} 1} \mathrm{EXXHX}_{\mathrm{n} 2} \mathrm{EX}_{\mathrm{n} 3} \mathrm{EXXH}$, which is predicted to 
be a di-iron center ligand (Stenmark et al. 2001). Alignment of $\operatorname{Coq} 7^{\mathrm{Xc}}$ and $\mathrm{Coq} 7$ identified a similar di-iron-binding motif. Conversion of $\mathrm{Coq} 7^{\mathrm{Xc}}$ key residues E96, E180, or H183 to alanine resulted in decreased CoQ8 biosynthesis (Fig. 4A), and similar analyses showed that residues G65, C68, and G75 are also essential for $\mathrm{Coq} 7^{\mathrm{Xc}}$ enzymatic activity (Fig. 4A). Recently, Busso et al. (2015) showed that Coq7 residues R57, V111, and S114 modulate Coq7 enzymatic activity. Among them, S114 was proposed as a new site for Coq7 phosphorylation. Sequence alignment analysis showed that the counterparts of the above three Coq7 residues in $\mathrm{Coq} 7^{\mathrm{Xc}}$ are R60, S112, and S115. Although point mutation of R60 and S115 significantly reduced Coq7 ${ }^{\mathrm{Xc}}$ 's activity for CoQ8 biosynthesis, point mutation of $\mathrm{S} 112$ had no effect (Fig. 4A).

Using the crystalline structure of di-iron carboxylate proteins and the proposed model of Coq7 (Busso et al. 2015; Frolow et al. 1994), we created a $\operatorname{Coq} 7^{X c}$ molecular model integrating information from our mutagenesis analysis with in silico analysis of residue conservation. The model consists of a fourhelix bundle, with helix 2 and 3 connected by a mostly disordered loop containing a small helix segment (Fig. 4B and C).

\section{Xc_0233 $\left(u b i J^{X c}\right)$ is required for CoQ8 biosynthesis in $X$. campestris pv. campestris.}

Genomic analysis of $X$. campestris pv. campestris strain 8004 revealed that the stop codon of Xc_0233 overlaps with the start codon of $u b i B^{\mathrm{Xc}}$, similar to what is found with $u b i J$ and $u b i B$ in E. coli. Although Xc_0233 and E. coli UbiJ have low aminoacid identity (21.8\%) (Supplementary Fig. S6), both contain a similar sterol carrier protein 2 (SCP2) at their N-terminal regions (Fig. 5A). These results suggest that Xc_0233 might have E. coli UbiJ-like activity. Thus, we refer to Xc_0233 as $u b i J^{\mathrm{Xc}}$ hereafter in this study.

During aerobic growth in $\mathrm{LB}$ medium at $28^{\circ} \mathrm{C}$, the $u b i J^{\mathrm{Xc}}$ deletion mutant $\Delta u b i J^{\mathrm{Xc}}$ exhibited a significant growth defect compared with the WT strain (Fig. 5B). Near-normal growth was restored to strain $\Delta u b i J^{\mathrm{Xc}}$ by introduction of a plasmid carrying $u b i J^{\mathrm{Xc}}$ (Fig. 5B). The cellular CoQ8 content of strain $\Delta u b i J^{\mathrm{Xc}}$ was $37.5 \%$ of that found in the WT strain (54.8 and $145.9 \mathrm{pmol} / \mathrm{mg}$ wet weight, respectively). Overexpression of the plasmid-borne $u b i J^{\mathrm{Xc}}$ in strain $\Delta u b i J^{\mathrm{Xc}}$ restored the CoQ8 content to WT levels (Fig. $5 \mathrm{C}$ and D). Because $u b i B^{\mathrm{Xc}}$ is located downstream of $u b i J^{\mathrm{Xc}}$ in $X$. campestris pv. campestris (Fig. 5A), we transformed the $u b i J^{\mathrm{Xc}}$ mutant with plasmids carrying $u b i B^{X c}$ or the $u b i J^{\mathrm{Xc}}-u b i B^{\mathrm{Xc}}$ gene cluster to determine the effect of the $u b i J^{\mathrm{Xc}}$ mutation on $u b i B^{\mathrm{Xc}}$ expression. Overexpression of $u b i B^{\mathrm{Xc}}$ had little effect on CoQ8 biosynthesis in strain $\Delta u b i J^{\mathrm{Xc}}$ (Fig. 6A and B), ruling out the possibility of any polar effect of the $u b i J^{\mathrm{Xc}}$ mutation. In contrast, overexpression of the $u b i J^{\mathrm{Xc}}-u b i B^{\mathrm{Xc}}$ gene cluster in strain $\Delta u b i J^{\mathrm{Xc}}$ restored CoQ8 to WT levels (Fig. 6B). Collectively, these results indicate that $u b i J^{\mathrm{Xc}}$ is required for CoQ8 biosynthesis in $X$. campestris pv. campestris.

Previously, Aussel et al. (2014a) showed that the $50 \mathrm{C}$ terminal amino-acid region of UbiJ was sufficient to sustain CoQ8 biosynthesis and normal aerobic growth of the E. coli ubiJ mutant strain. In this study, partial $\mathrm{UbiJ}^{\mathrm{Xc}}$ regions covering the 54 and $80 \mathrm{C}$-terminal amino acids or the $135 \mathrm{~N}$-terminal amino acids were overexpressed in the $X$. campestris pv. campestris ubiJ ${ }^{\mathrm{Xc}}$ mutant. Although the overexpression of the 80 C-terminal amino-acid region did restore bacterial growth and CoQ8 biosynthesis (Fig. 5B and C), the 135 N-terminal amino-acid and the $54 \mathrm{C}$-terminal amino-acid regions had no significant effect.

In an attempt to further verify that $X$. campestris pv. campestris $\mathrm{UbiJ}^{\mathrm{Xc}}$ plays a role similar to that of $E$. coli UbiJ in CoQ8 biosynthesis, cross-complementation analyses with
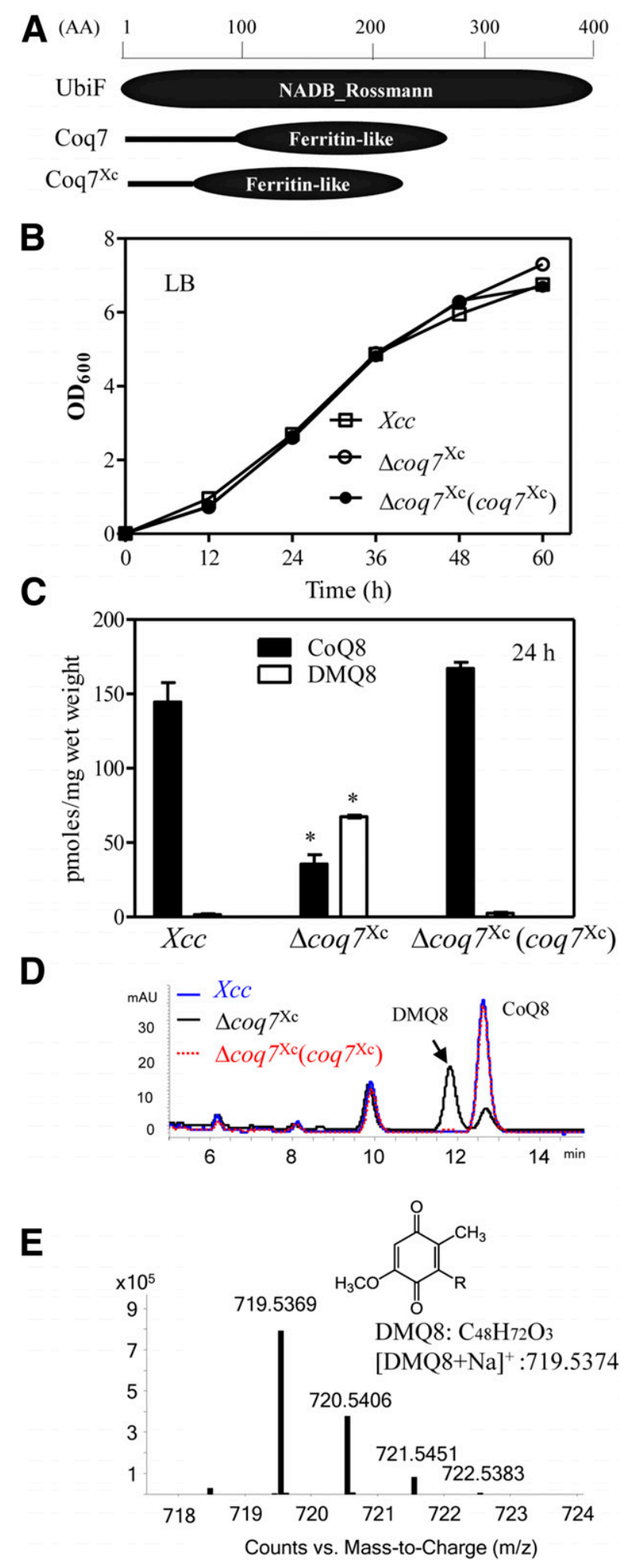

Fig. 2. Xc_0489 $\left(\operatorname{coq} 7^{\mathrm{Xc}}\right)$ is required for coenzyme Q8 (CoQ8) biosynthesis in Xanthomonas campestris pv. campestris. A, Domain organization of UbiF, $\mathrm{CoQ} 7^{\mathrm{Xc}}$, and Coq7 of Saccharomyces cerevisiae. B, Growth curves of $X$. campestris pv. campestris $(X c c)$ strains in Luria-Bertani (LB) medium. $\mathrm{OD}_{600}=$ optical density at $600 \mathrm{~nm}$. C, Quantitative analysis of CoQ8 and demethoxy-Q8 (DMQ8) biosynthesis by X. campestris pv. campestris cultures at $24 \mathrm{~h}$ after inoculation. D, High-performance liquid chromatography analysis of CoQ8 and DMQ8 biosynthesis in X. campestris pv. campestris strains. E, Verification of the identity of DMQ8 found in the $\Delta \operatorname{coq} 7^{\mathrm{Xc}}$ culture by ultrahigh performance liquid chromatography tandem mass spectrometry. Averages for three independent experiments with standard deviations are shown. Statistically significant differences with respect to the $X$. campestris pv. campestris strain are indicated by one asterisk $(P \leq 0.05)$. 


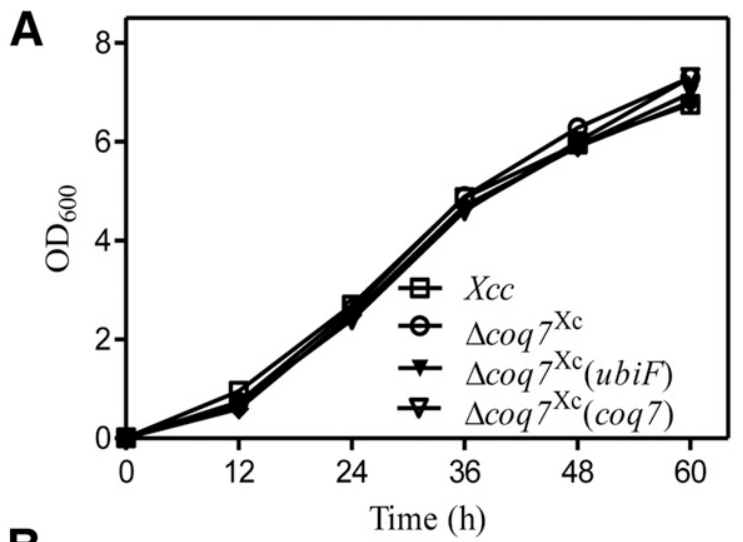

B
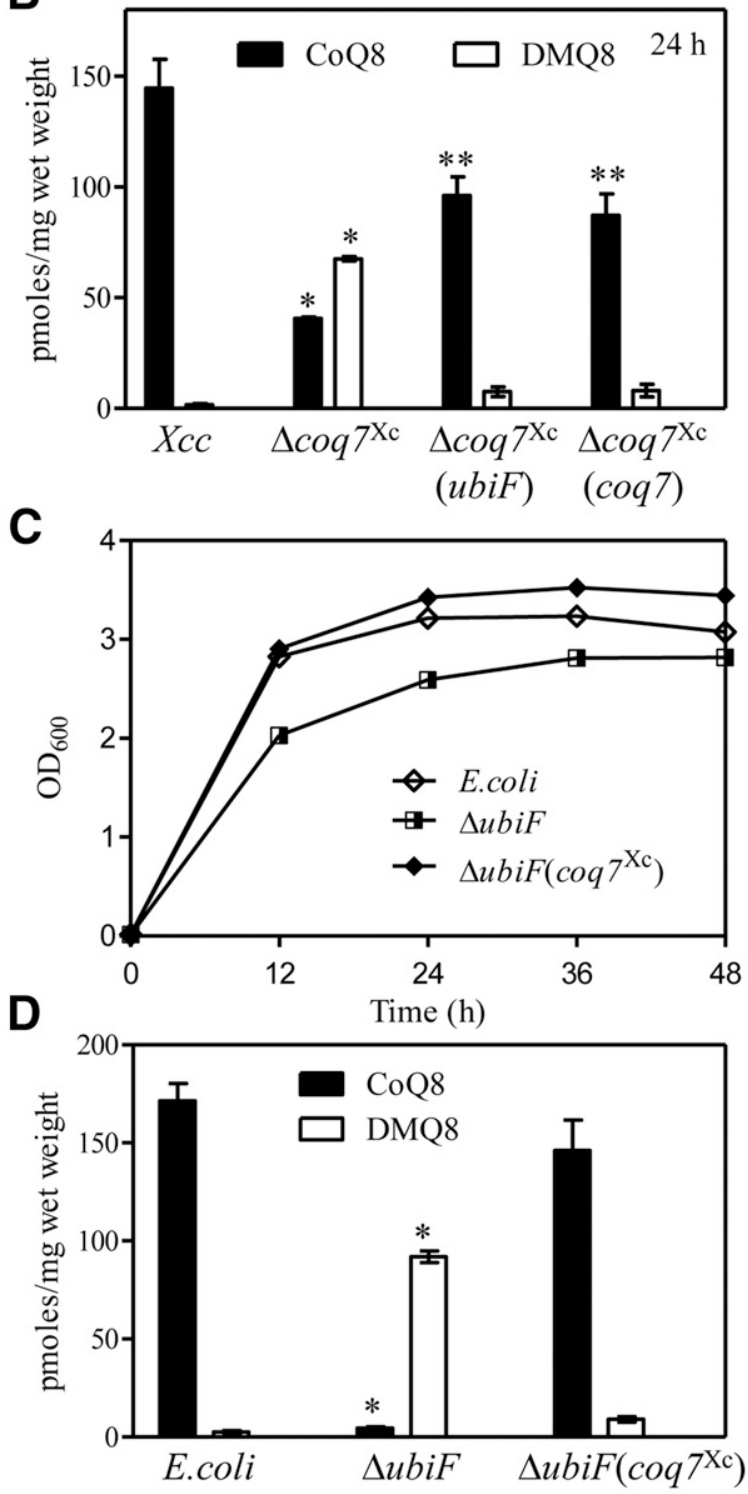

Fig. 3. Complementation analysis of Xanthomonas campestris pv. campestris strains $\Delta \operatorname{coq} 7^{\mathrm{Xc}}$ and $\Delta u b i F$. A, Growth curves of $X$. campestris pv. campestris strains. B, Biosynthesis of coenzyme Q8 (CoQ8) and demethoxy-Q8 (DMQ8) by X. campestris pv. campestris strains at an optical density at $600 \mathrm{~nm}\left(\mathrm{OD}_{600}\right)=1.6$ in LB medium. C, Growth curves of Escherichia coli strains. D, Biosynthesis of CoQ8 and DMQ8 by E. coli strains at $\mathrm{OD}_{600}=1.6$. Averages for three independent experiments with standard deviation are shown. Statistically significant differences with respect to the wild-type strains of $X$. campestris pv. campestris or E. coli are indicated by one asterisk $(P \leq 0.05)$.
$X$. campestris pv. campestris strain $\Delta u b i J^{\mathrm{Xc}}$ or $E$. coli strain $\Delta u b i J$ were conducted. Although overexpression of $u b i J^{\mathrm{Xc}}$ or the $u b i J^{\mathrm{Xc}}-u b i B^{\mathrm{Xc}}$ gene cluster in the $X$. campestris pv. campestris strain $\Delta u b i J^{\mathrm{Xc}}$ fully restored bacterial growth and CoQ8 biosynthesis to WT levels (Figs. 5B and $\mathrm{C}$ and $6 \mathrm{~A}$ and $\mathrm{B}$ ), overexpression of E. coli ubiJ or the ubiJ-ubiB gene cluster in the same $X$. campestris pv. campestris strain had no effect (Fig. $6 \mathrm{~A}$ and B). Similarly, although overexpression of $u b i J$ or the $u b i J-u b i B$ gene cluster in the $E$. coli strain $\Delta u b i J$ fully restored bacterial growth and CoQ8 biosynthesis (Fig. 6C and D), overexpression of $u b i J^{\mathrm{Xc}}$ or the $u b i J^{\mathrm{Xc}}-u b i B^{\mathrm{Xc}}$ gene cluster in this strain had no significant effect (Fig. 6C and D).

\section{$X$. campestris pv. campestris $\Delta u b i J^{\mathrm{Xc}}$ cells} accumulate the intermediate OHB.

High-performance liquid chromatography (HPLC) analysis of the $\Delta u b i J^{\mathrm{Xc}}$ strain CoQ extracts detected the accumulation of an unknown electroactive compound which was not present in the CoQ extracts of the WT X. campestris pv. campestris strain (Fig. $5 \mathrm{C}$ and D). Overexpression of $u b i J^{\mathrm{Xc}}$ in the mutant strain
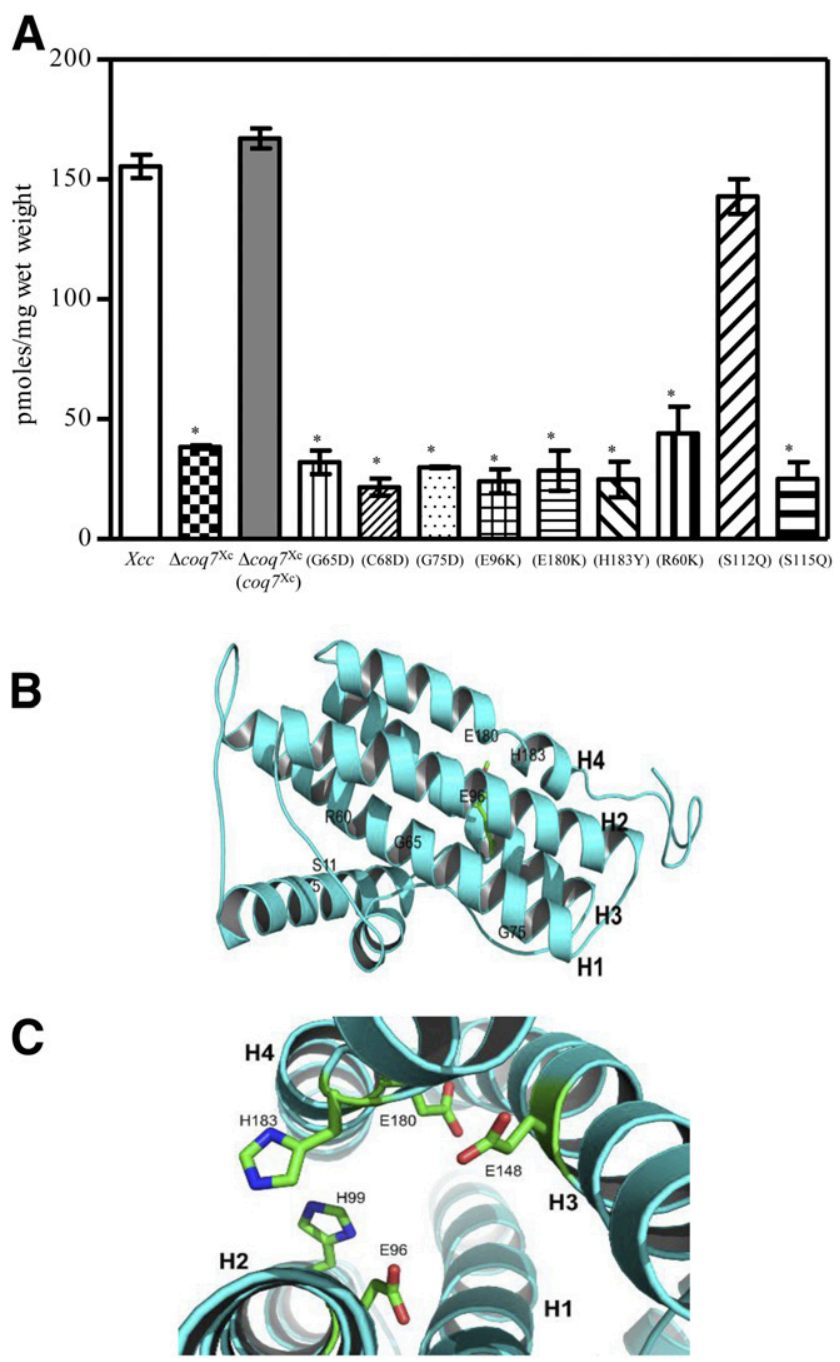

Fig. 4. Point mutation and molecular modeling analyses indicate that $\mathrm{Coq} 7^{\mathrm{Xc}}$ is a di-iron carboxylate monooxygenase with a four-helix bundle. A, Coenzyme Q $(\mathrm{CoQ})$ production of Xanthomonas campestris pv. campestris strains mutated at key $\mathrm{CoQ} 7^{\mathrm{Xc}}$ residues associated with catalytic activity. $\mathbf{B}, \mathrm{CoQ} 7^{\mathrm{Xc}}$ model showing a four-helix bundle and mutagenized residues. C, Di-iron center residues and their location inside helices. Averages for three independent experiments with standard deviation are shown. Statistically significant differences with respect to the wild-type $X$. campestris pv. campestris strain are indicated by one asterisk $(P \leq 0.05)$. 


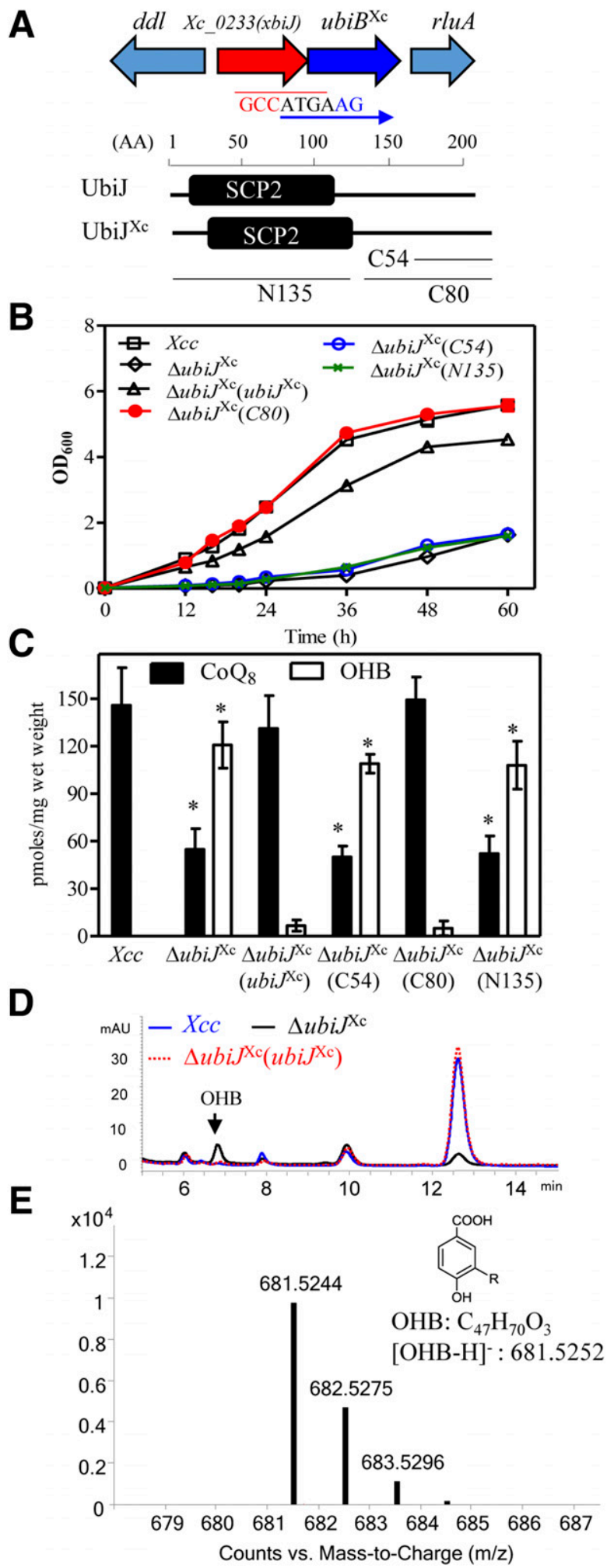

Fig. 5. Xc_0233 is required for coenzyme Q8 (CoQ8) biosynthesis in Xanthomonas campestris pv. campestris. A, Genomic localization of $u b i J^{\mathrm{Xc}}$ in $X$. campestris pv. campestris strain 8004 and domain organization of $\mathrm{UbiJ}^{\mathrm{Xc}}$ and UbiJ. B, Growth curves of $X$. campestris pv. campestris strains in Luria-Bertani (LB) medium. $\mathrm{OD}_{600}=$ optical density at $600 \mathrm{~nm}$. C, Quantitative analysis of CoQ8 and 3-octaprenyl-4-hydroxybenzoic acid (OHB) biosynthesis in $X$. campestris pv. campestris strains. D, High-performance liquid chromatography analyses of CoQ8 and $\mathrm{OHB}$ biosynthesis in $X$. campestris pv. campestris strains at $\mathrm{OD}_{600}$ in LB medium. $\mathbf{E}$, Verification of the identity of $\mathrm{OHB}$ found in strain $\Delta u b i J^{X c}$ by ultrahigh-performance liquid chromatography tandem mass spectrometry. Averages for three independent experiments with standard deviation are shown. Statistically significant differences with respect to the strain $X$. campestris pv. campestris are indicated by one asterisk $(P \leq 0.05)$.
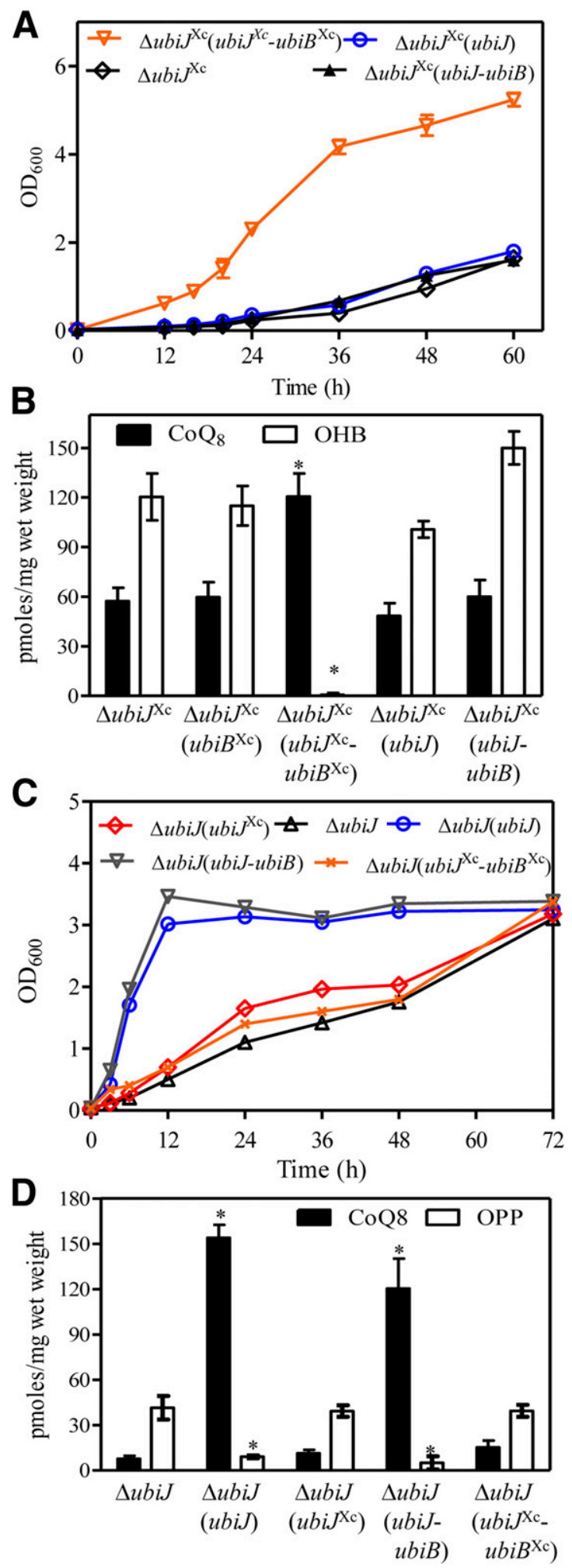

Fig. 6. Complementation analysis of Xanthomonas campestris pv. campestris strains $\Delta u b i J^{X c}$ and $\Delta u b i J$. A, Growth curves of X. campestris pv. campestris strains in Luria-Bertani (LB) medium. $\mathrm{OD}_{600}=$ optical density at $600 \mathrm{~nm}$. B, Quantitative analysis of coenzyme Q8 (CoQ8) and 3octaprenyl-4-hydroxybenzoic acid (OHB) biosynthesis in X. campestris pv. campestris strains at $\mathrm{OD}_{600}=1.6$. C, Growth curves of Escherichia coli strains in LB medium. D, Quantitative analysis of Coq8 and 3-octaprenylphenol (OPP) biosynthesis in E. coli strains at $\mathrm{OD}_{600}=1.6$. Averages for three independent experiments with standard deviation are shown. Statistically significant differences with respect to the strain $X$. campestris pv. campestris are indicated by one or two asterisks $(P \leq 0.05$ or 0.01 , respectively). 


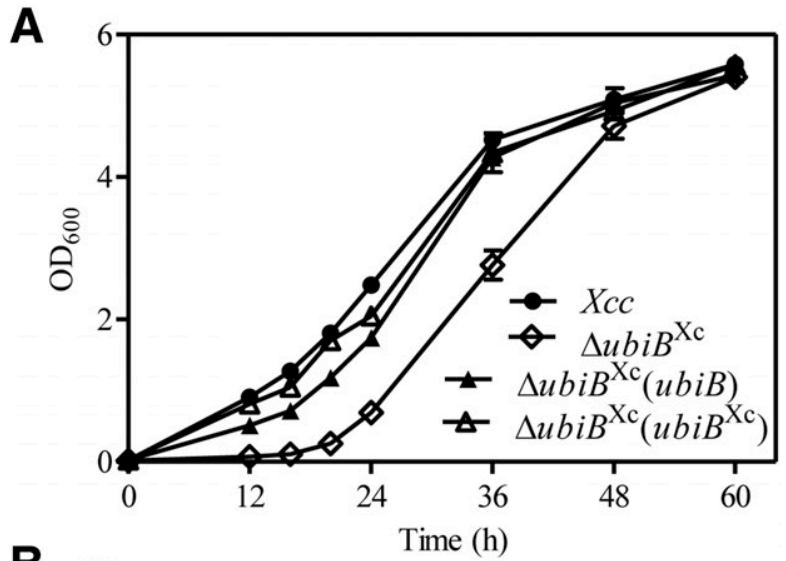

B
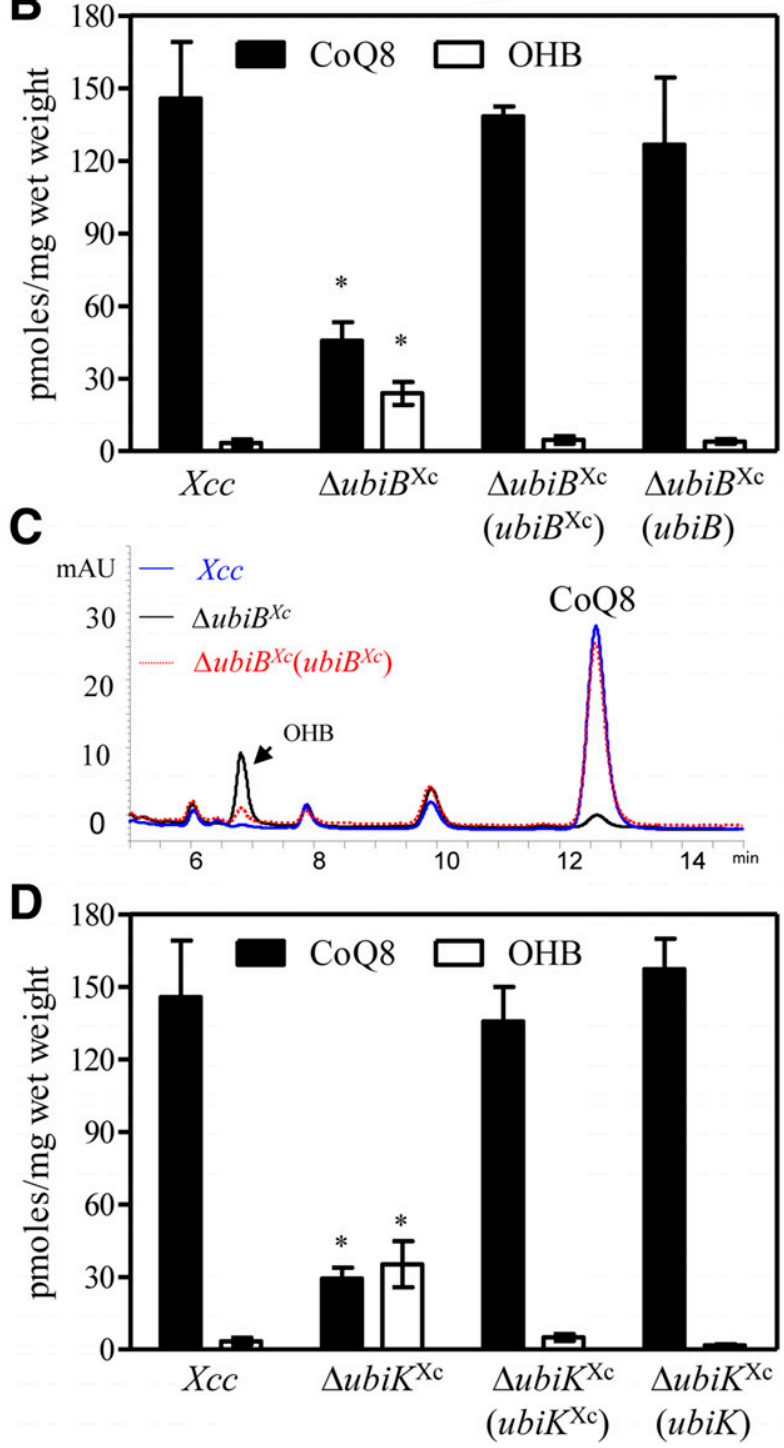

Fig. 7. Deletion of Xanthomonas campestris pv. campestris $u b i B^{\mathrm{Xc}}$ or $u b i K^{\mathrm{Xc}}$ significantly decreased coenzyme Q8 (CoQ8) biosynthesis, leading to accumulation of the intermediate 3-octaprenyl-4-hydroxybenzoic acid (OHB). A, Growth time course of $X$. campestris pv. campestris-derived strains. $\mathrm{OD}_{600}=$ optical density at $600 \mathrm{~nm}$. B, Quantitative analysis of $\mathrm{CoQ} 8$ and $\mathrm{OHB}$ biosynthesis in $\triangle u b i B^{\mathrm{Xc}}$-derived cultures at $\mathrm{OD}_{600}=1.6 . \mathbf{C}$, High-performance liquid chromatography analysis of CoQ8 and OHB biosynthesis in $X$. campestris pv. campestris-derived strains. D, Quantitative analysis of CoQ8 and $\mathrm{OHB}$ production in $\Delta u b i K^{\mathrm{Xc}}$-derived cultures at $\mathrm{OD}_{600}=$ 1.6. Averages for three independent experiments with standard deviations are shown. Statistically significant differences with respect to the strain $X$. campestris pv. campestris are indicated by one asterisk $(P \leq 0.05)$.
$\Delta u b i J^{\mathrm{Xc}}$ almost completely eliminated accumulation of this compound. Further analysis of the unknown compound by UPLC-MS in positive mode gave an $\mathrm{m} / \mathrm{z}$ ratio $\left(\mathrm{M}+\mathrm{H}^{+}\right)$of 681.5244, which corresponded to a chemical formula of $\mathrm{C}_{47} \mathrm{H}_{70} \mathrm{O}_{3}(\mathrm{MW}=681.5252)$ (Fig. 5E). Our unpublished data showed that fragmentation of the 681.5 molecular ion yielded a characteristic tropylium-like ion. This is often found for aromatic compounds containing a benzyl unit. Taken together, the unknown compound should be one of the CoQ biosynthetic intermediates, OHB, in E. coli (Fig. 5E).

We then generated the $u b i J$ in-frame deletion mutant $\Delta u b i J$ in WT E. coli strain MG1655. HPLC analysis of the $\triangle u b i J$ CoQ extract subsequently revealed a new electroactive compound not found in the WT strain. Further MS analysis indicated that this compound is the biosynthetic intermediate 3-octaprenylphenol (OPP), which has previously been shown to accumulate in E. coli $\triangle u b i B$ and $\triangle u b i K$ strains (Loiseau et al. 2017; Poon et al. 2000). Overexpression of ubiJ in strain $\Delta u b i J$ abolished the accumulation of OPP. No OPP was found to accumulate in the $X$. campestris pv. campestris $\Delta u b i J^{\mathrm{Xc}}$ cells (Fig. 5D) and no OHB was found to accumulate in the E. coli $\Delta u b i J$ cells.

$u b i B^{\mathrm{Xc}}$ and $u b i K^{\mathrm{Xc}}$ are involved

in $X$. campestris pv. campestris CoQ8 biosynthesis.

$X$. campestris pv. campestris UbiB (encoded by Xc_0234, denoted as $u b i B^{\mathrm{Xc}}$ hereafter) and E. coli UbiB have $47 \%$ aminoacid identity and a similar domain organization. Deletion of $u b i B^{\mathrm{Xc}}$ both significantly decreased CoQ8 biosynthesis and led to an accumulation of $\mathrm{OHB}$ in $X$. campestris pv. campestris (Fig. 7B and C). Subsequently, WT levels of CoQ8 and OHB were restored to the $\Delta u b i B^{\mathrm{Xc}}$ mutant by overexpressing $u b i B^{\mathrm{Xc}}$ or E. coli ubiB (Fig. 7B and C).

$X$. campestris pv. campestris $\mathrm{UbiK}^{\mathrm{Xc}}$ and E. coli $\mathrm{UbiK}$ have $50 \%$ amino-acid identity and have a similar domain organization. As with $u b i B^{\mathrm{Xc}}$, deletion of $u b i K^{\mathrm{Xc}}$ also resulted in significantly decreased CoQ8 biosynthesis and an accumulation of $\mathrm{OHB}$ (Fig. 7D). Likewise, overexpression of $u b i K^{\mathrm{Xc}}$ or $E$. coli $u b i K$ restored CoQ8 and OHB to WT levels (Fig. 7D).

In previous studies, the biosynthetic intermediate OPP accumulated to high levels in E. coli $\triangle u b i B$ and $\triangle u b i K$ strains (Loiseau et al. 2017; Poon et al. 2000). In this study, mutant strain $\triangle u b i B$ was generated in E. coli strain MG1655, and decreased CoQ8 biosynthesis and significant OPP accumulation were observed. Subsequently, CoQ8 and OPP were restored to WT levels by plasmids carrying either $u b i B$ or $u b i B^{\mathrm{Xc}}$ (Supplementary Fig. S7).

$\mathrm{UbiK}^{\mathrm{Xc}}$ interacts with $\mathrm{UbiJ}^{\mathrm{Xc}}$ and $\mathrm{UbiB}^{\mathrm{Xc}}$

in $X$. campestris pv. campestris.

Yeast two-hybrid (Y2H) analysis was conducted in $X$. campestris pv. campestris to detect possible interactions between $\mathrm{UbiK}^{\mathrm{Xc}}$, $\mathrm{UbiJ}^{\mathrm{Xc}}$, and $\mathrm{UbiB}^{\mathrm{Xc}}$. There were no detectable protein level interactions between $\mathrm{UbiB}^{X c}$ and the $80 \mathrm{C}$-terminal amino-acid region of either $\mathrm{UbiJ}^{\mathrm{Xc}}$ or $\mathrm{UbiJ}^{\mathrm{Xc}}$ (Supplementary Fig. S8).

However, further investigations revealed strong binding between $\mathrm{UbiK}^{\mathrm{Xc}}$ and $\mathrm{UbiJ}^{\mathrm{Xc}}$, and between $\mathrm{UbiK}^{\mathrm{Xc}}$ and $\mathrm{UbiB}^{\mathrm{Xc}}$. No interactions were detected between $\mathrm{UbiK}^{\mathrm{Xc}}$ and either E. coli UbiJ or E. coli UbiB.

The genes $\operatorname{coq} 7^{\mathrm{Xc}}, u b i B^{\mathrm{Xc}}, u b i K^{\mathrm{Xc}}$, and $u b i J^{\mathrm{Xc}}$ are associated with virulence in Chinese radish.

By using the scissor clipping method of inoculation, as previously described (He et al. 2006), we showed that the lesion lengths of CoQ8 mutant strains $\Delta \operatorname{coq} 7^{\mathrm{Xc}}, \Delta u b i J^{\mathrm{Xc}}, \Delta u b i B^{\mathrm{Xc}}$, and $\Delta u b i K^{\mathrm{Xc}}$ on Chinese radish were significantly less than that of the WT strain X. campestris pv. campestris (Fig. 8). 


\section{DISCUSSION}

Biosynthesis of $\mathrm{CoQ}$ in $X$. campestris pv. campestris via a yeast-like pathway?

In E. coli, the products of the $u b i A, u b i D, u b i E, u b i F, u b i G$, $u b i H, u b i I$, and $u b i X$ genes catalyze the functionalization of the phenyl ring of the 4-HBA precursor, including prenylation, decarboxylation, hydroxylation, and methylation reactions (Aussel et al. 2014a; Hajj Chehade et al. 2013; Kawamukai 2016; White et al. 2015). All enzymatic reactions necessary for Q8 biosynthesis in aerobic conditions were assigned to the corresponding Ubi proteins. The findings in this study suggest that the catalytic reactions in $X$. campestris pv. campestris CoQ8 biosynthetic pathway are generally similar to those in E. coli. First, $X$. campestris pv. campestris and E. coli have seven genes ( $u b i A, u b i B, u b i E, u b i G, u b i H, u b i I$, and $u b i K)$ in common. Individual deletion of four of them (ubiB, ubiH, ubiI, and $u b i K)$ resulted in either the full or partial elimination of CoQ8 biosynthesis (Fig. 7). Second, although XanB2 and $\operatorname{Coq} 7^{\mathrm{Xc}}$ in $X$. campestris pv. campestris are completely different from UbiC and UbiF in amino acid size and domain organization, they have similar catalytic activities. $x a n B 2$ was able to complement E. coli $\triangle u b i C$ for CoQ8 biosynthesis (Zhou et al. 2013a and b). $\Delta \operatorname{coq} 7^{\mathrm{Xc}}$ and $\Delta u b i F$ strains could be genetically crosscomplemented by either $\operatorname{coq} 7^{\mathrm{Xc}}$ or $u b i F$ (Fig. 3). In addition, $X$. campestris pv. campestris $\Delta \operatorname{coq} 7^{\mathrm{Xc}}$ cells and E. coli $\Delta u b i F$ cells accumulated the same biosynthetic intermediate, DMQ8 (Fig. 2).

However, the present study also suggests that the order of the catalytic reactions in $X$. campestris pv. campestris CoQ8 biosynthesis might be different from that of E. coli. Decarboxylation is one of the keys steps in the bacterial CoQ biosynthetic pathway. In E. coli, $u b i D$ and $u b i X$ are responsible for the decarboxylation step (Payne et al. 2015; White et al. 2015). However, no homologs of $u b i D$ and $u b i X$ were identified in $X$. campestris pv. campestris (Fig. 1). Similar observations were also made for the CoQ6 biosynthetic pathway of $S$. cerevisiae-no gene for decarboxylation was identified (Kawamukai 2016). A comparison of the CoQ biosynthetic pathways in E. coli and $S$. cerevisiae shows that they diverge after the assembly of the common intermediate OHB (González-Mariscal et al. 2014; Kawamukai 2016). E. coli CoQ8 biosynthesis proceeds by decarboxylation of OHB followed by oxidation and O-methylation. In contrast, the $S$. cerevisiae CoQ6 biosynthetic pathway proceeds with oxidation of $\mathrm{OHB}$, followed by O-methylation and then decarboxylation. Because both X. campestris pv. campestris and $S$. cerevisiae use the same type of C6-monooxygenase (Coq $7^{\mathrm{Xc}}$ and Coq7, respectively) in CoQ biosynthesis, and no decarboxylase was identified in either strain, it is tempting to propose that $X$. campestris pv. campestris might use a S. cerevisiae-like order of catalytic reactions for CoQ8 biosynthesis. The decarboxylation stage of the ring modification procedure could be after hydroxylation and methylation in X. campestris pv. campestris. This idea is further supported by the findings that deletion of $u b i J^{\mathrm{Xc}}, u b i B^{\mathrm{Xc}}$, and $u b i K^{\mathrm{Xc}}$ resulted in an accumulation of the intermediate $\mathrm{OHB}$ in $X$. campestris pv. campestris (Figs. 5, 6, and 7) while deletion of the E. coli gene counterparts led to an accumulation of OPP.

\section{Divergent regulatory mechanisms of CoQ biosynthesis} in $X$. campestris pv. campestris?

Several lines of evidence indicate that UbiK, UbiJ, and UbiB may form a complex involved in the regulation of $E$. coli $\mathrm{CoQ} 8$ biosynthesis. First, although the products of $u b i B$, ubiJ, and $u b i K$ are required for the efficient biosynthesis of CoQ8, none of them is predicted to carry out an enzymatic function (Hajj
Chehade et al. 2013; Lagier-Tourenne et al. 2008; Poon et al. 2000). Second, inactivation of any one of these genes drastically reduces CoQ8 levels but this leads to the accumulation of only the early intermediate OPP, which is not diagnostic of a defect in any specific biosynthetic step. Third, the UbiK protein physically interacts with UbiJ and several other Ubi proteins (Loiseau et al. 2017). X. campestris pv. campestris contains homologs of $E$. coli $u b i B$ and $u b i K$, and a homolog of $E$. coli $u b i J$ $\left(u b i B^{X c}, u b i K^{X c}\right.$, and $u b i J^{\mathrm{Xc}}$, respectively) (Fig. 1). All three of these genes are required for efficient CoQ8 biosynthesis in $X$. campestris pv. campestris (Figs. 5 and 7). $u b i B^{\mathrm{Xc}}$ and $u b i K^{\mathrm{Xc}}$ can complement the E. coli mutant strains $\triangle u b i B$ and $\Delta u b i K$, respectively, and vice versa (Fig. 7). $\mathrm{UbiK}^{\mathrm{Xc}}$ also interacts with $\mathrm{UbiJ}^{\mathrm{Xc}}$ and $\mathrm{UbiB}^{\mathrm{Xc}}$ in $X$. campestris pv. campestris. All of these findings support the idea that the $\mathrm{UbiK}^{\mathrm{Xc}}$ complex may play roles similar to those of the E. coli UbiK complex to regulate CoQ8 biosynthesis in $X$. campestris pv. campestris.

However, the present study also suggests that the UbiK complex might act on different reaction steps of the CoQ8 biosynthetic pathway in $X$. campestris pv. campestris and E. coli. First, deletion of $u b i B^{X c}, u b i J^{\mathrm{Xc}}$, or $u b i K^{\mathrm{Xc}}$ led to the accumulation of OHB in X. campestris pv. campestris (Figs. 5 and 7), whereas deletion of $u b i B, u b i J$, or $u b i K$ in $E$. coli resulted in the accumulation of OPP (Loiseau et al. 2017; Poon et al. 2000). In the proposed E. coli CoQ8 biosynthetic pathway, $\mathrm{OHB}$ is a product of UbiA while OPP is a product of OHB decarboxylation. Second, although both $\mathrm{UbiJ}^{\mathrm{Xc}}$ and $E$. coli UbiJ contain the same SCP2 domain, E. coli ubiJ could not complement the $X$. campestris pv. campestris $\Delta u b i J^{\mathrm{Xc}}$ strain and vice versa (Fig. 6). There can be several reasons for this. Codon usage bias may cause a protein level difference in both $E$. coli and $X$. campestris pv. campestris. UbiJ ${ }^{\mathrm{Xc}}$ may require additional unknown factors for its optimal activity or conformation in $X$. campestris pv. campestris. Our further results also indicate that this is probably due to the fact that $\mathrm{UbiK}^{\mathrm{Xc}}$ interacts with $\mathrm{UbiJ}^{\mathrm{Xc}}$ but has no binding affinity to $E$. coli UbiJ. Finally, overexpression of E. coli $u b i B$ in the E. coli strain $\triangle u b i B$ diminished OPP accumulation to WT levels whereas overexpression of $u b i B$ in the $X$. campestris pv. campestris strain $\Delta u b i B^{\mathrm{Xc}}$ diminished OHB accumulation to WT levels (Fig. 7B). This finding suggests that $X$. campestris pv. campestris UbiJ $^{\mathrm{Xc}}$ and $E$. coli UbiJ may exert their roles in strain-specific manners. The roles of UbiK complex in E. coli and X. campestris pv. campestris CoQ biosynthesis deserve further investigation.

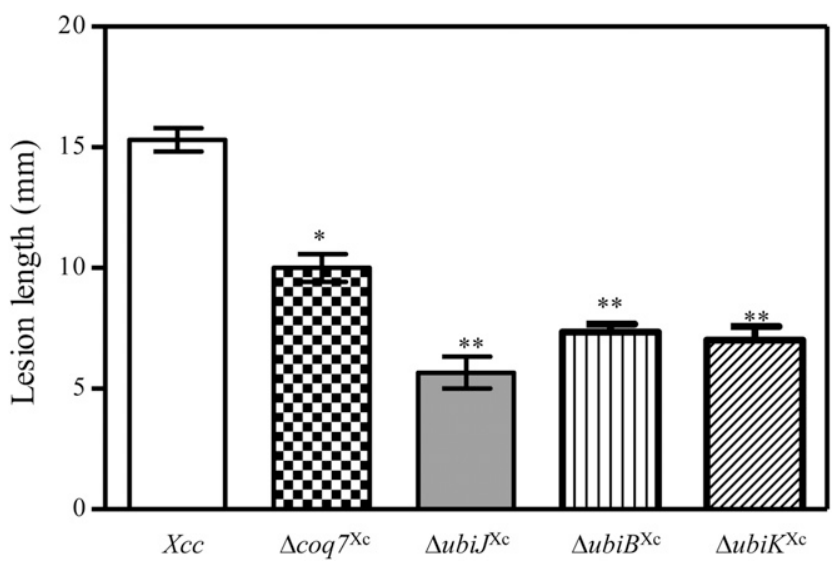

Fig. 8. Lesion length of Xanthomonas campestris pv. campestris-derived mutant strains $\Delta \operatorname{coq} 7^{\mathrm{Xc}}, \Delta u b i J^{\mathrm{Xc}}, \Delta u b i B^{\mathrm{Xc}}$, and $\Delta u b i K^{\mathrm{Xc}}$ in Chinese radish. Averages for three independent experiments with standard deviations are shown. Statistically significant differences with respect to the $X$. campestris pv. campestris wild-type strain are indicated by one or two asterisks $(P \leq$ 0.05 or 0.01 , respectively). 


\section{Significance of CoQ biosynthetic gene diversity.}

Previously, the gene Rv2949c was characterized as a novel chorismate lyase responsible for the direct conversion of chorismate to 4-HBA in Mycobacterium tuberculosis (Stadthagen et al. 2005). Pelosi et al. (2016) conducted a nice phylogenomic analysis of hydroxylases (UbiF, $\mathrm{UbiH}$, and UbiI) involved in $\mathrm{CoQ}$ biosynthesis. Their results revealed that proteobacteria evolved a surprising diversity in the CoQ hydroxylase repertoire. In the present study, although the gene for OHB decarboxylase remains unknown, three unique genes $\left(x a n B 2, \operatorname{coq} 7^{\mathrm{Xc}}\right.$, and $u b i J^{X c}$ ) were characterized to be required for CoQ8 biosynthesis in the phytopathogen $X$. campestris pv. campestris. All of these findings suggest a genetic diversity in CoQ8 biosynthesis between $X$. campestris pv. campestris and E. coli.

All organisms exist in environments that vary in time and space, and genetic diversity allows populations to adapt to changing environments (Frankham 2005). The more genetic diversity a population has, the greater the likelihood that the population will be able to adapt to changing circumstances (Pullin 2002). Both X. campestris pv. campestris and E. coli have different life styles. E. coli is a facultatively anaerobic bacterium and is commonly found in the lower intestine of warm-blooded animals. It makes ATP by aerobic respiration in the presence of oxygen but is capable of switching to fermentation or anaerobic respiration if oxygen is absent (Tenaillon et al. 2010). In contrast, $X$. campestris pv. campestris is a strict aerobe which is known to cause black rot in crucifers (Leyns et al. 1984). It can persist in soil or on plant surfaces for over a year. As a vascular pathogen, $X$. campestris pv. campestris can also multiply inside plants and colonize their xylem elements (Wang et al. 2015). Thus, the question of whether other new CoQ biosynthetic genes and the putative divergent biosynthetic pathway in X. campestris pv. campestris are associated with their lifestyles deserves further investigation. Much remains to be done to fully understand the sophisticated molecular mechanisms.

The CoQ biosynthetic pathway decarboxylase gene has not yet been identified in many of the Gram-negative bacteria, $S$. cerevisiae, and all higher organisms. Because $X$. campestris pv. campestris and S. cerevisiae share similar CoQ biosynthetic pathways, $X$. campestris pv. campestris should be a new model organism for the study of $\mathrm{CoQ}$ biosynthesis, particularly the unknown gene for decarboxylation.

\section{MATERIALS AND METHODS}

\section{Bacterial strains and growth conditions.}

$X$. campestris pv. campestris and E. coli strains (Supplementary Table S1) were grown in LB broth with shaking (200 rpm) at 28 and $37^{\circ} \mathrm{C}$, respectively. When required, antibiotics were added to a final concentration of kanamycin at $50 \mathrm{mg} /$ liter, rifampicin at $25 \mathrm{mg} / \mathrm{liter}$, ampicillin at $100 \mathrm{mg} / \mathrm{liter}$, tetracycline at $5 \mathrm{mg} / \mathrm{liter}$, and gentamycin at $10 \mathrm{mg} / \mathrm{liter}$. Solid media contained 2\% agar (Sangon Biotech, Shanghai, China).

\section{Gene deletion and functional complementation analysis.}

$X$. campestris pv. campestris strain 8004 in-frame deletion mutants were generated using the methods described previously (He et al. 2006). E. coli gene deletion was conducted following the method previously described (Datsenko and Wanner 2000). Primers used for gene deletion are listed in Supplementary Table S2. Mutant complementation analyses were conducted by either of two methods. First, the coding region of each gene was amplified by polymerase chain reaction (PCR) and cloned into the expression vector pBBR-1-MCS $\left(\operatorname{Kan}^{\mathrm{R}}\right)$ (Kovach et al. 1995). The resulting constructs were transferred to $X$. campestris pv. campestris strains through triparental mating. Alternatively, the target gene together with its promoter was amplified by PCR, then cloned into the vector pUC18-miniTn7T-Gm. The target gene was subsequently integrated into the $X$. campestris pv. campestris chromosome using the methods previously described (Jittawuttipoka et al. 2009).

In silico genome analysis and bioinformatic analysis. The whole genome of $X$. campestris pv. campestris strain 8004 was downloaded from the National Center for Biotechnology Information (NCBI), and the sequences of $u b i A,-B,-C,-D,-E,-F$, $-G,-H,-I,-J,-K$, and $-X$ were downloaded from the genome of $E$. coli strain MG1655. The BLASTP program from NCBI was then used to identify homologs of $E$. coli $u b i$ genes in the genome of $X$. campestris pv. campestris strain 8004. The homologs were selected based on two major criteria: (i) a threshold amino-acid identity of $25 \%$ and (ii) similar aminoacid size. Multiple sequence alignment analysis was performed using Clustal W, and all the nucleotide segments were translated using GENSCAN.

\section{Site-directed mutation of the gene $\operatorname{coq} 7^{\mathrm{Xc}}$.}

$\operatorname{coq} 7^{\mathrm{Xc}}$ was cloned into pGEM-T-Easy vector (Promega Corp., Madison, WI, U.S.A.), and the QuikChange SiteDirected Mutagenesis Kit was used to obtain $\operatorname{coq} 7^{\mathrm{Xc}}$ point mutations. These were then verified by sequence analysis and subsequently cloned into the expression vector pBBR-1-MCS $\left(\operatorname{Kan}^{\mathrm{R}}\right)$. In the final step of the analysis, the resulting constructs were transferred to the $\Delta \operatorname{coq} 7^{\mathrm{Xc}}$ strain through triparental mating.

\section{$\operatorname{Coq} 7^{\mathrm{Xc}}$ molecular modeling.}

The $\operatorname{Coq} 7^{\mathrm{Xc}}$ primary sequence was obtained from the NCBI database. Molecular modeling was performed with the primary sequence as input data for protein threading in I-Tasser (Zhang 2008). All runs were performed using Auto Dock 4.0 (Morris et al. 2009), with global search exhaustiveness set to 20 and conformer maximum number set to 10. Among docking poses, lower binding energy conformations were selected for DMQ1. Figures were generated using PyMOL (DeLano 2002).

\section{CoQ extraction, purification, and quantification by HPLC analysis.}

CoQ was extracted following the previously described protocol (Zhou et al. 2013a). Briefly, 50-ml LB cultures of $X$. campestris pv. campestris strains were grown to an optical density (OD) of 1.6, and cells were then collected by centrifugation. Cell pellets were resuspended in $2 \mathrm{ml}$ of $0.2 \mathrm{M}$ acetate buffer (pH 5.6) after washing twice with $1 \times$ phosphate-buffered saline (PBS) buffer. Cryo-impacted cell homogenates were subsequently subjected to sonication for a total of $90 \mathrm{~s}$, and $5 \mathrm{ml}$ of hexane-acetone $(1: 1, \mathrm{vol} / \mathrm{vol})$ reaction mixture was added to each cell homogenate, followed by sonication and vortexing. The hexane fractions were collected, and the solvent was removed by rotary evaporation to dryness at $40^{\circ} \mathrm{C}$. The residues were then dissolved in $1.5 \mathrm{ml}$ of chloroform-methanol $(1: 1$, $\mathrm{vol} / \mathrm{vol}$ ), followed by washing with $0.75 \mathrm{ml}$ of $0.7 \% \mathrm{NaCl}$. The chloroform fractions were then collected and solvent was removed by rotary evaporation to dryness at $40^{\circ} \mathrm{C}$. These residues were then dissolved in $100 \mu \mathrm{l}$ of methanol each, and the crude extracts were further analyzed by HPLC (Agilent 1260 Infinity; Agilent Technologies, Palo Alto, CA, U.S.A.). The area under each chromatographic peak was integrated and quantified. A standard curve for CoQ8 was created by plotting the peak area versus the known concentrations. The concentration of CoQ8 in the extract was calculated based on the peak area and the standard curve. The Coq8 content in X. campestris pv. campestris was finally converted as picomoles per milligram of wet weight according to the same amount of cells taken for each measurement. 


\section{UPLC-MS analysis and quantification of CoQ8, DMQ8, OPP, and OHB.}

Eluate containing the purified CoQ8 was subjected to electrospray ionization (ESI)-MS analysis on an Agilent 6230 TOF MS equipped with a Jet Stream ESI source (Agilent Technologies), using $100 \%$ methanol containing $1.5 \%$ formic acid as mobile phase. Briefly, the column temperature was maintained at $30^{\circ} \mathrm{C}$ and the flow rate was kept at $0.4 \mathrm{ml} / \mathrm{min}$. In total, $5 \mu \mathrm{l}$ of CoQ8 standard solutions or $5 \mu \mathrm{l}$ of the extract was injected into a 1290 Infinity UPLC equipped with a Zorbax Eclipse XDBC18 column. The signals were detected in a diode array detector at a wavelength of $220 \mathrm{~nm}$ with a bandwidth of $\pm 4 \mathrm{~nm}$. The UPLC elutes were further introduced into an accurate mass TOF-MS in the negative ionization mode and equipped with a Jet Stream ESI source (Agilent Technologies). The area under each chromatographic peak was integrated and quantified. A standard curve for CoQ8 was created by plotting the peak area versus the known concentrations. The concentration of CoQ8 in the extract was calculated based on the peak area and the standard curve.

\section{Y2H assay.}

$\mathrm{Y} 2 \mathrm{H}$ assays were performed using the Matchmaker Gold Yeast Two-Hybrid System (Clontech, Mountain View, CA, U.S. A.). The test gene fragment was cloned from $X$. campestris $\mathrm{pv}$. campestris strain 8004 by PCR amplification and ligated into the prey vector pGADT7 or bait vector pGBKT7. The resulting constructs were transformed into S. cerevisiae AH109 according to the manual instructions, and transformants were selected on a synthetic defined (SD) medium lacking leucine and tryptophan (SD-Leu-Trp). These strains were then suspended in $1 \times$ PBS buffer, and four 10-fold serial dilutions were placed on SD medium lacking Leu, Trp, and histidine (SD-Leu-Trp-His). Protein interactions were screened on SD medium lacking Leu, Trp, His, and adenine (SD-Leu-Trp-His-Ade). Growth of the yeast cells on SD-Leu-Trp-His-Ade plates in 2 days indicated strong interactions between the test proteins.

\section{Virulence assay in host plant.}

For the virulence assay, bacterial pellets from cultures harvested at an OD at $600 \mathrm{~nm}=1.6$ were resuspended in sterile PBS buffer to an OD of 0.05 , then inoculated into adult radish plants by leaf clipping. Lesion lengths were measured 14 days after inoculation. Fifteen leaves were inoculated for each independent experiment, and each treatment was repeated three times.

\section{Statistical analysis.}

All experiments were performed at least three times. Analysis of variance for experimental data sets was performed using JMP software (version 5.0; SAS Institute Inc., Cary, NC, U.S.A.). Significant effects of treatment were determined by the $F$ value $(P=0.05)$. When a significant $F$ test was obtained, separation of means was accomplished by Fisher's protected least significant difference at $P=0.05$. The investigators were blinded to the group allocation by randomly selecting single colonies multiple times. No statistical methods were used to predetermine sample size.

\section{LITERATURE CITED}

Alcázar-Fabra, M., Navas, P., and Brea-Calvo, G. 2016. Coenzyme Q biosynthesis and its role in the respiratory chain structure. Biochim. Biophys. Acta 1857:1073-1078.

Aussel, L., Loiseau, L., Hajj Chehade, M., Pocachard, B., Fontecave, M., Pierrel, F., and Barras, F. 2014a. ubiJ, a new gene required for aerobic growth and proliferation in macrophage, is involved in coenzyme Q biosynthesis in Escherichia coli and Salmonella enterica serovar Typhimurium. J. Bacteriol. 196:70-79.
Aussel, L., Pierrel, F., Loiseau, L., Lombard, M., Fontecave, M., and Barras, F. 2014b. Biosynthesis and physiology of coenzyme Q in bacteria. Biochim. Biophys. Acta 1837:1004-1011.

Bentinger, M., Tekle, M., and Dallner, G. 2010. Coenzyme Q-Biosynthesis and functions. Biochem. Biophys. Res. Commun. 396:74-79.

Busso, C., Ferreira-Júnior, J. R., Paulela, J. A., Bleicher, L., Demasi, M., and Barros, M. H. 2015. Coq7p relevant residues for protein activity and stability. Biochimie 119:92-102.

Datsenko, K. A., and Wanner, B. L. 2000. One-step inactivation of chromosomal genes in Escherichia coli K-12 using PCR products. Proc. Natl. Acad. Sci. U.S.A. 97:6640-6645.

DeLano, W. L. 2002. The PyMol Molecular Graphics System. DeLano Scientific, San Carlos, CA

Frankham, R. 2005. Genetics and Extinction. Biol. Conserv. 126:131-140.

Frolow, F., Kalb, A. J., and Yariv, J. 1994. Structure of a unique twofold symmetric haem-binding site. Nat. Struct. Biol. 1:453-460.

González-Mariscal, I., García-Testón, E., Padilla, S., Martín-Montalvo, A., Pomares Viciana, T., Vazquez-Fonseca, L., Gandolfo Domínguez, P., and Santos-Ocaña, C. 2014. The regulation of coenzyme q biosynthesis in eukaryotic cells: All that yeast can tell us. Mol. Syndromol. 5:107-118.

Hajj Chehade, M., Loiseau, L., Lombard, M., Pecqueur, L., Ismail, A., Smadja, M., Golinelli-Pimpaneau, B., Mellot-Draznieks, C., Hamelin, O., Aussel, L., Kieffer-Jaquinod, S., Labessan, N., Barras, F., Fontecave, M., and Pierrel, F. 2013. ubiI, a new gene in Escherichia coli coenzyme $\mathrm{Q}$ biosynthesis, is involved in aerobic C5-hydroxylation. J. Biol. Chem. 288:20085-20092.

He, Y. W., Xu, M., Lin, K., Ng, Y. J., Wen, C. M., Wang, L. H., Liu, Z. D., Zhang, H. B., Dong, Y. H., Dow, J. M., and Zhang, L. H. 2006. Genome scale analysis of diffusible signal factor regulon in Xanthomonas campestris pv. campestris: Identification of novel cell-cell communicationdependent genes and functions. Mol. Microbiol. 59:610-622.

Jittawuttipoka, T., Buranajitpakorn, S., Fuangthong, M., Schweizer, H. P., Vattanaviboon, P., and Mongkolsuk, S. 2009. Mini-Tn7 vectors as genetic tools for gene cloning at a single copy number in an industrially important and phytopathogenic bacteria, Xanthomonas spp. FEMS Microbiol. Lett. 298:111-117.

Kawamukai, M. 2016. Biosynthesis of coenzyme Q in eukaryotes. Biosci. Biotechnol. Biochem. 80:23-33.

Kovach, M. E., Elzer, P. H., Hill, D. S., Robertson, G. T., Farris, M. A., Roop, R. M., 2nd, and Peterson, K. M. 1995. Four new derivatives of the broad-host-range cloning vector pBBR1MCS, carrying different antibioticresistance cassettes. Gene 166:175-176.

Kwon, O., Kotsakis, A., and Meganathan, R. 2000. Ubiquinone (coenzyme Q) biosynthesis in Escherichia coli: Identification of the $u b i F$ gene. FEMS Microbiol. Lett. 186:157-161.

Lagier-Tourenne, C., Tazir, M., López, L. C., Quinzii, C. M., Assoum, M., Drouot, N., Busso, C., Makri, S., Ali-Pacha, L., Benhassine, T., Anheim, M., Lynch, D. R., Thibault, C., Plewniak, F., Bianchetti, L., Tranchant, C., Poch, O., DiMauro, S., Mandel, J. L., Barros, M. H., Hirano, M., and Koenig, M. 2008. ADCK3, an ancestral kinase, is mutated in a form of recessive ataxia associated with coenzyme Q10 deficiency. Am. J. Hum. Genet. 82:661-672.

Lee, P. T., Hsu, A. Y., Ha, H. T., and Clarke, C. F. 1997. A Cmethyltransferase involved in both ubiquinone and menaquinone biosynthesis: Isolation and identification of the Escherichia coli ubiE gene. J. Bacteriol. 179:1748-1754.

Letunic, I., Doerks, T., and Bork, P. 2015. SMART: Recent updates, new developments and status in 2015. Nucleic Acids Res. 43:D257-D260.

Leyns, F., De Cleene, M., Swings, J. G., and De Ley, J. 1984. The host range of the genus Xanthomonas. Bot. Rev. 50:308-356

Loiseau, L., Fyfe, C., Aussel, L., Hajj Chehade, M., Hernández, S. B., Faivre, B., Hamdane, D., Mellot-Draznieks, C., Rascalou, B., Pelosi, L., Velours, C., Cornu, D., Lombard, M., Casadesús, J., Pierrel, F., Fontecave, M., and Barras, F. 2017. The UbiK protein is an accessory factor necessary for bacterial ubiquinone (UQ) biosynthesis and forms a complex with the UQ biogenesis factor UbiJ. J. Biol. Chem. 292: 11937-11950.

Mansfield, J., Genin, S., Magori, S., Citovsky, V., Sriariyanum, M., Ronald, P., Dow, M., Verdier, V., Beer, S. V., Machado, M. A., Toth, I., Salmond, G., and Foster, G. D. 2012. Top 10 plant pathogenic bacteria in molecular plant pathology. Mol. Plant Pathol. 13:614-629.

Marbois, B. N., and Clarke, C. F. 1996. The COQ7 gene encodes a protein in saccharomyces cerevisiae necessary for ubiquinone biosynthesis J. Biol. Chem. 271:2995-3004.

Morris, G. M., Huey, R., Lindstrom, W., Sanner, M. F., Belew, R. K., Goodsell, D. S., and Olson, A. J. 2009. AutoDock4 and AutoDockTools4: Automated docking with selective receptor flexibility. J. Comput. Chem. 30:2785-2791. 
Okada, K., Kamiya, Y., Zhu, X., Suzuki, K., Tanaka, K., Nakagawa, T., Matsuda, H., and Kawamukai, M. 1997. Cloning of the $s d s A$ gene encoding solanesyl diphosphate synthase from Rhodobacter capsulatus and its functional expression in Escherichia coli and Saccharomyces cerevisiae. J. Bacteriol. 179:5992-5998.

Payne, K. A., White, M. D., Fisher, K., Khara, B., Bailey, S. S., Parker, D., Rattray, N. J., Trivedi, D. K., Goodacre, R., Beveridge, R., Barran, P., Rigby, S. E., Scrutton, N. S., Hay, S., and Leys, D. 2015. New cofactor supports $\alpha, \beta$-unsaturated acid decarboxylation via 1,3-dipolar cycloaddition. Nature 522:497-501.

Pelosi, L., Ducluzeau, A. L., Loiseau, L., Barras, F., Schneider, D., Junier, I., and Pierrel, F. 2016. Evolution of ubiquinone biosynthesis: Multiple proteobacterial enzymes with various regioselectivities to catalyze three contiguous aromatic hydroxylation reactions. mSystems 1:e00091-e16.

Poon, W. W., Barkovich, R. J., Hsu, A. Y., Frankel, A., Lee, P. T., Shepherd, J. N., Myles, D. C., and Clarke, C. F. 1999. Yeast and rat Coq3 and Escherichia coli UbiG polypeptides catalyze both O-methyltransferase steps in coenzyme Q biosynthesis. J. Biol. Chem. 274:21665-21672.

Poon, W. W., Davis, D. E., Ha, H. T., Jonassen, T., Rather, P. N., and Clarke, C. F. 2000. Identification of Escherichia coli ubiB, a gene required for the first monooxygenase step in ubiquinone biosynthesis. J. Bacteriol. 182:5139-5146.

Pullin, A. S. 2002. Conservation Biology, 1st ed. Cambridge University Press, Cambridge.

Sharma, P., Teixeira de Mattos, M. J., Hellingwerf, K. J., and Bekker, M. 2012. On the function of the various quinone species in Escherichia coli. FEBS J. 279:3364-3373.

Stadthagen, G., Korduláková, J., Griffin, R., Constant, P., Bottová, I., Barilone, N., Gicquel, B., Daffé, M., and Jackson, M. 2005. pHydroxybenzoic acid synthesis in Mycobacterium tuberculosis. J. Biol. Chem. 280:40699-40706.

Stefely, J. A., Reidenbach, A. G., Ulbrich, A., Oruganty, K., Floyd, B. J., Jochem, A., Saunders, J. M., Johnson, I. E., Minogue, C. E., Wrobel, R. L., Barber, G. E. Lee, D., Li, S., Kannan, N., Coon, J. J., Bingman, C. A., and Pagliarini, D. J. 2015. Mitochondrial ADCK3 employs an atypical protein kinase-like fold to enable coenzyme Q biosynthesis. Mol. Cell. 57:83-94.

Stenmark, P., Grünler, J., Mattsson, J., Sindelar, P. J., Nordlund, P., and Berthold, D. A. 2001. A new member of the family of di-iron carboxylate proteins. Coq7 (clk-1), a membrane-bound hydroxylase involved in ubiquinone biosynthesis. J. Biol. Chem. 276:33297-33300.

Tenaillon, O., Skurnik, D., Picard, B., and Denamur, E. 2010. The population genetics of commensal Escherichia coli. Nat. Rev. Microbiol. 8:207-217.

Wang, J. Y., Zhou, L., Chen, B., Sun, S., Zhang, W., Li, M., Tang, H., Jiang, B. L., Tang, J. L., and He, Y. W. 2015. A functional 4-hydroxybenzoate degradation pathway in the phytopathogen Xanthomonas campestris is required for full pathogenicity. Sci. Rep. UK 5:18456.

White, M. D., Payne, K. A., Fisher, K., Marshall, S. A., Parker, D., Rattray, N. J., Trivedi, D. K., Goodacre, R., Rigby, S. E., Scrutton, N. S., Hay, S. and Leys, D. 2015. UbiX is a flavin prenyltransferase required for bacterial ubiquinone biosynthesis. Nature 522:502-506.

Wu, G., Williams, H. D., Zamanian, M., Gibson, F., and Poole, R. K 1992. Isolation and characterization of Escherichia coli mutants affected in aerobic respiration: The cloning and nucleotide sequence of $u b i G$. Identification of an S-adenosylmethionine-binding motif in protein, RNA, and small-molecule methyltransferases. J. Gen. Microbiol. 138:2101-2112.

Zhang, Y. 2008. I-TASSER server for protein 3D structure prediction. BMC Bioinf. 9:40-47.

Zhou, L., Huang, T. W., Wang, J. Y., Sun, S., Chen, G., Poplawsky, A., and He, Y. W. 2013a. The rice bacterial pathogen Xanthomonas oryzae pv. oryzae produces 3-hydroxybenzoic acid and 4-hydroxybenzoic acid via $\mathrm{XanB} 2$ for use in xanthomonadin, ubiquinone, and exopolysaccharide biosynthesis. Mol. Plant-Microbe Interact. 26:1239-1248.

Zhou, L., Wang, J.-Y., Wu, J., Wang, J., Poplawsky, A., Lin, S., Zhu, B., Chang, C., Zhou, T., Zhang, L. H., and He, Y. W. 2013b. The diffusible factor synthase $\mathrm{XanB} 2$ is a bifunctional chorismatase that links the shikimate pathway to ubiquinone and xanthomonadins biosynthetic pathways. Mol. Microbiol. 87:80-93.

\section{AUTHOR-RECOMMENDED INTERNET RESOURCES}

National Center for Biotechnology Information: https://www.ncbi.nlm.nih.gov/

GENSCAN: http://genes.mit.edu/GENSCAN.html 